\title{
Tratamento cirúrgico do pneumotórax espontâneo primário no primeiro episódio*
}

\author{
Darcy Ribeiro Pinto Filho ${ }^{1}$, André Germano Leite ${ }^{2}$, Fabíola Délia Perin ${ }^{3}$, Ronaldo Barbieri ${ }^{3}$
}

\begin{abstract}
Objetivo: Avaliar os resultados do tratamento cirúrgico do PEP através da toracotomia axilar, ressecção de blebs e pleurodese abrasiva. Mediante os resultados e a comparação destes com as diversas opções de tratamento, discutir a abordagem cirúrgica como a primeira para os pacientes portadores de PEP, no primeiro episódio. Material e métodos: 35 toracotomias axilares com pleurodese abrasiva para tratamento do PEP foram realizadas no Serviço de Cirurgia Torácica da Universidade de Caxias do Sul entre janeiro de 1996 e março de 1999. Resultados: 23 pacientes foram tratados no primeiro episódio de PEP $(65,7 \%)$ e 12 pacientes no segundo episódio. Os drenos pleurais permaneceram em média $2,7 \pm 1,6$ dias. O período médio de internação foi de $4,9 \pm 2,4$ dias. Morbidade de 8,5\%, mortalidade zero. Não se evidenciou recidiva num período médio de 22 meses de acompanhamento. Conclusão: A indicação de toracotomia axilar com pleurodese abrasiva em pacientes portadores de pneumotórax espontâneo primário no primeiro ou segundo episódio mostrou baixos índices de morbidade, recidiva zero, além de curto período de permanência dos drenos pleurais e de hospitalização, comprovando sua eficácia terapêutica. A possibilidade de indicá-lo como primeiro método terapêutico para pacientes portadores de PEP deve ser considerada.
\end{abstract}

(J Pneumol 2001;27(3):153-157)

\section{Surgical treatment of primary spontaneous pneumothorax on the first episode}

Purpose: To assess the results of the surgical treatment of primary spontaneous pneumothorax $(P S P)$ by means of axillary thoracotomy, blebs resection and abrasive pleurodesis, by comparing the results with the different options of treatment, and to discuss the surgical approach as the best therapeutic option for patient with the first occurrence of PSP. Material and methods: 35 axillary thoracotomy with abrasive pleurodesis for the treatment of PSP were carried out at the Department of Thoracic Surgery of the University of Caxias do Sul between January 1996 and March 1999. Results: 23 patients with their first episode of PSP and 12 patients in their second episode were treated. The pleural drains were kept for in an average period of $2.7 \pm 1.6$ days. The average time in hospital was about $4.9 \pm 2.4$ days. Morbidity was $8.5 \%$ and mortality rate was zero. There was no relapse in an average period of 22 months of follow-up. Conclusion: The indication of axillary thoracotomy and abrasive pleurodesis to the patients with primary spontaneous pneumothorax (PSP) at the first or recurrent episode showed low rates of morbidity and no relapse, besides short periods of hospitalization and pleural drain permanence. The possibility of indication as the first therapeutic method for the PSP should be considered.

* Trabalho realizado no Serviço de Cirurgia Torácica da Universidade de Caxias do Sul.

1. Responsável pelo Serviço de Cirurgia Torácica; Professor de Cirurgia Torácica.

2. Cirurgião Torácico.

3. Acadêmico de Medicina.
Endereço para correspondência - Darcy R. Pinto Filho, Av. Abramo Randon, 1.201, apto. 72 - 95055-010 - Caxias do Sul, RS, Brasil. Fax (54) 222-6488; E-mail darcyrp@zaz.com.br

Recebido para publicação em 31/8/00. Reapresentado em 7 / 12/00. Aprovado, após revisão, em 15/12/00. 
Descritores - Pneumotórax. Toracotomia.

Key words - Pneumothorax. Thoracotomy.

\section{INTRODUÇÃO}

A literatura e a prática médica sedimentaram as indicações para o tratamento cirúrgico do pneumotórax espontâneo primário: segundo episódio, fuga aérea prolongada por mais de cinco dias, pneumotórax contralateral, pneumotórax simultâneo bilateral e, no primeiro episódio, em profissões de risco (mergulhadores, aviadores). No entanto, os avanços observados nas técnicas operatória e anestésica, a melhoria nos cuidados clínicos aos pacientes, aliados à necessidade de contenção dos custos terapêuticos, induzem à reflexão sobre a indicação do tratamento cirúrgico no primeiro episódio de pneumotórax espontâneo primário, para os casos não caracterizados nas indicações convencionais.

Os critérios que definiram as indicações atuais de tratamento cirúrgico do pneumotórax espontâneo primário foram estabelecidos nas décadas de 60 e $70^{(1,2)}$, quando não dispúnhamos dos recursos da medicina contemporânea. É necessário reconhecer a prudência das indicações cirúrgicas àquela época e a consciência sobre os riscos da indicação de métodos mais "agressivos" para o tratamento de uma patologia benigna que atinge uma população preponderantemente jovem.

Métodos menos agressivos, tais como a observação ou aspiração com agulha para os casos de pneumotórax pequenos (volumes $<20 \%$ ), ou mesmo a drenagem pleural para os de volume acima de $20 \%$, representam o tratamento convencional do pneumotórax espontâneo primário no primeiro episódio, com probabilidade de recidiva que varia entre $25 \%$ e $60 \%$ em várias séries da literatura $^{(3-5)}$.

Os trabalhos surgidos a partir da década de 80 e 90 trazem os resultados do tratamento cirúrgico através de toracotomia mínima (toracotomia axilar) e mesmo a videotoracoscopia, com índice zero de mortalidade, mínima morbidade, recidiva inferior a $4 \%$ e menor período de internação hospitalar(6-10).

A questão que se impõe é por que não oferecê-los como primeira opção de tratamento para os casos de pneumotórax espontâneo primário no primeiro episódio?

\section{MATERIAL E MÉTODOS}

No período de janeiro de 1996 a março de 1999 foram analisados 35 pacientes submetidos à toracotomia axilar com pleurodese abrasiva para tratamento de pneumotórax espontâneo primário (PEP), no Serviço de Cirur-
Siglas e abreviaturas utilizadas neste trabalho

PEP - Pneumotórax espontâneo primário

PES - Pneumotórax espontâneo secundário

TCT - Tomografia computadorizada de tórax

PNT - Pneumotórax

gia Torácica da Universidade de Caxias do Sul. Nos casos de PEP no segundo episódio, a indicação foi formal. Para os portadores de PEP, no primeiro episódio, foram discutidos os índices de morbidade e recidiva com os pacientes, comparando drenagem simples e toracotomia axilar, para que houvesse o consentimento ao procedimento proposto. Período de internação hospitalar, permanência dos drenos pleurais, índices de recidiva, complicações perioperatórias, profissão, idade, volume de pneumotórax e associação com o hábito tabágico foram avaliados.

O diagnóstico definitivo foi estabelecido através de radiografias simples de tórax de frente e perfil, assim como o volume do pneumotórax, estimado através da mensuração da distância entre o ápice do pulmão e do hemitórax em centímetros, extrapolando-se esta medida para um percentual correspondente (ex.: $3 \mathrm{~cm}-30 \%$ ) - proposto por Rhea et al. ${ }^{(11)}$ como alternativa à sua fórmula que calcula as médias das distâncias intrapleurais e estabelece o volume de pneumotórax correspondente.

Nenhum dos pacientes realizou tomografia computadorizada com intuito de auxiliar o diagnóstico ou mesmo identificar bolhas ou blebs contralaterais.

Todos foram submetidos à toracotomia axilar longitudinal, em média, de $5 \mathrm{~cm}$, junto ao cavum axilar, abordando a cavidade através do $2^{\circ}$ ou $3^{\circ}$ espaço intercostal. A inspeção da cavidade pleural objetivou principalmente o ápice pulmonar para identificação de blebs subpleurais. Nos casos de dúvida quanto à existência de outras bolhas, utilizamos o recurso da pleuroscopia como método auxiliar. A ressecção das blebs apicais foi realizada sob clamp vascular e envolveu pequena faixa de parênquima pulmonar apical, suturado com categute cromado 3-0 em pontos aero e hemostáticos. Escarificação com gaze seca até observarmos sufusão hemorrágica em toda a pleura parietal (pleurodese abrasiva). Não realizamos abrasão sobre a pleura visceral. Teste do "borracheiro" (pulmão submerso em soro fisiológico e submetido à pressão positiva pelo anestesista) para verificar a efetividade da aerostasia da sutura pulmonar e drenagem com dreno $\mathrm{n}^{\circ} 26$ logo abaixo da linha da toracotomia.

O dreno foi retirado após 24 horas de cessar eventual fuga aérea e a demonstração da expansibilidade pulmonar através da radiografia de controle. No $30^{\circ}$ dia de pósoperatório os pacientes foram submetidos à revisão clínico-radiológica. Os pacientes foram contatados por telefone ou carta durante os últimos dois meses do estudo. 


\section{Resultados}

A idade dos pacientes variou entre 14 e 57 anos, com média de 28 anos. Observou-se preponderância do sexo masculino (77,1\%). O hábito tabágico esteve presente em $45,7 \%$ dos casos. Dor torácica de início súbito em todos os casos, dispnéia em $74,3 \%$ e tosse em $22,9 \%$ foram os principais sintomas relatados pelos pacientes. O esforço físico associado ao início dos sintomas foi referido por três pacientes $(8,6 \%)$. Pneumotórax à esquerda em 20 pacientes, 15 casos à direita, nenhum caso de pneumotórax espontâneo bilateral concomitante. $\mathrm{O}$ volume do pneumotórax, aferido pela radiografia simples de tórax, variou entre $20 \%$ e $80 \%$. Nenhum paciente referiu profissão de risco para desenvolvimento de pneumotórax espontâneo.

Vinte e três pacientes foram tratados cirurgicamente (toracotomia axilar) no primeiro episódio. Dois destes pacientes apresentaram indicação formal de cirurgia no primeiro episódio: hemotórax em um caso e fuga aérea prolongada em outro. Os restantes 12 pacientes foram operados em decorrência do segundo episódio de pneumotórax espontâneo primário. Nenhum caso de terceiro

TABELA 1

Características clínicas de 35 pacientes submetidos à toracotomia axilar com pleurodese abrasiva

\begin{tabular}{lc}
\hline \multicolumn{1}{c}{ Variável } & Freqüência \\
\hline Sexo & \\
Masculino & $27(77,1 \%)$ \\
Feminino & $8(22,9 \%)$ \\
Idade & $28(14$ a 57$)$ \\
Média & $16(45,7 \%)$ \\
Tabagismo & \\
Sintomas & $35(100 \%)$ \\
Dor torácica & $26(74,3 \%)$ \\
Dispnéia & $8(22,9 \%)$ \\
Tosse & $3(8,6 \%)$ \\
Esforço físico & \\
Hemitórax afetado & $15(42,9 \%)$ \\
Direito & $20(57,1 \%)$ \\
Esquerdo & $2,7 \pm 1,6$ dias \\
Permanência dos drenos (total $)$ & $2,8 \pm 1,8$ dias \\
$1^{\circ}$ episódio $(\mathrm{n}=23)$ & $2,4 \pm 1,1 \mathrm{dias}$ \\
$2^{\circ}$ episódio $(\mathrm{n}=12)$ & $4,9 \pm 2,4$ dias \\
Hospitalização & $5,1 \pm 2,4$ dias \\
$1^{\circ}$ episódio $(\mathrm{n}=23)$ & $4,8 \pm 2,1 \mathrm{dias}$ \\
$2^{\circ}$ episódio $(\mathrm{n}=12)$ & $3(8,5 \%)$ \\
Complicações & Zero \\
Mortalidade & Zero \\
Recidiva & \\
\hline
\end{tabular}

episódio foi documentado. A cirurgia torácica videoassistida não foi utilizada nesta série de pacientes operados.

Os drenos pleurais permaneceram em média $2,7 \pm 1,6$ dias. O período médio de internação hospitalar foi de 4,9 $\pm 2,4$ dias. Houve um caso de hemotórax, dois casos de seroma e deiscência da sutura cutânea intradérmica, abrigando um índice de complicações de $8,5 \%$. Mortalidade zero e segmento médio de 22 meses dos casos operados. Não identificamos recidiva na série citada (Tabela 1).

A análise estatística (teste $t$ de Student) comparando os dois grupos de pacientes submetidos à toracotomia axilar no primeiro episódio $(\mathrm{n}=23)$ e no segundo episódio $(\mathrm{n}=$ 12), no que se refere à efetividade do método, avaliada através das médias de permanência dos drenos pleurais, período de hospitalização e recidiva, não mostrou diferença estatisticamente significante $(p=0,736)$, para um intervalo de confiança de $95 \%$ entre -1,479 e 2,073 (Tabela 2).

\section{DISCUSSÃO}

Os objetivos terapêuticos principais no pneumotórax espontâneo são o restabelecimento da negatividade pleural, promoção da expansão pulmonar total e prevenção da recorrência. Secundariamente, a terapêutica deve apresentar mínima morbidade, além de tempo de internação e custos reduzidos.

A clássica publicação de Seremetis ${ }^{(2)}$, na qual 155 pacientes portadores de pneumotórax espontâneo primário foram estudados, estabeleceu as bases para a atual proposta terapêutica. Índices de recidiva de $49 \%$ para pacientes tratados com repouso no leito, $40 \%$ para os tratados com repouso seguido de drenagem pleural, $38 \%$ para os tratados inicialmente com drenagem e recidiva zero para os tratados com toracotomia e pleurodese foram documentados nessa série. A demonstração de menores indices de recorrência para os paciente tratados com drenagem sustentou a indicação deste método para a pri-

TABELA 2

Análise comparativa dos grupos de pacientes submetidos à toracotomia axilar no primeiro e segundo episódios de pneumotórax espontâneo primário

\begin{tabular}{lccc}
\hline & Drenos pleurais & Hospitalização & Recidiva \\
\hline $\begin{array}{l}\text { Toracotomia } \\
\text { axilar } 1^{\circ} \text { episódio } \\
(\mathrm{n}=23)\end{array}$ & $2,8 \pm 1,8$ dias & $5,1 \pm 2,5$ dias & zero \\
$\begin{array}{l}\text { Toracotomia } \\
\text { axilar } 2^{\circ} \text { episódio } \\
(\mathrm{n}=12)\end{array}$ & $2,4 \pm 1,1$ dias & $4,8 \pm 2,1$ dias & zero \\
\hline
\end{tabular}

Teste $t$ de Student; $\mathrm{p}=0,736 ; \mathrm{IC}=95 \%(-1,479$ a 2,073$)$ 
meira ocorrência de pneumotórax espontâneo primário, nos pacientes que apresentassem câmaras de pneumotórax com valor estimado superior a $20 \%$ da cavidade pleural.

Com exceção dos que exerçam profissões de risco, como mergulhadores e aviadores, pacientes com fuga aérea persistente ou pneumotórax contralateral, a maioria é tratada com drenagem pleural. Alguns poucos são observados ou submetidos à punção aspirativa com agulha, mediante avaliação do volume do pneumotórax $(<20 \%)$ e estabilidade clínica. As indicações de cirurgia, basicamente, recaem para os casos de primeira recorrência em diante e para as exceções do primeiro episódio ${ }^{(7,12)}$.

Mesmo com o surgimento de alternativas técnicas, como a toracotomia axilar ou videotoracoscopia, e a melhoria dos cuidados clínicos e anestésicos, não se observou qualquer proposta de modificação das indicações de intervenção cirúrgica no PEP.

Os índices de recidiva ou recorrência após o primeiro episódio, em pacientes tratados com observação, punção com agulha ou drenagem, variam de 20 a $60 \%{ }^{(3-5)}$ e os fatores que predispõem a esta recorrência ainda não estão bem estabelecidos. Um estudo conduzido por Sadikot et al. ${ }^{(12)}$ em 153 pacientes portadores de pneumotórax espontâneo primário tratados através de drenagem pleural, com seguimento de quatro anos (recidiva de 54,2\%), analisou as variáveis idade, sexo, peso e altura, volume do pneumotórax, hábito tabágico e terapêutica inicial, na tentativa de identificar eventuais fatores preditivos de recidiva. A avaliação estatística através de análise multivariada e regressão logística concluiu que os índices de recorrência não estavam relacionados ao peso, altura, tratamento inicial ou volume do pneumotórax $(p<0,01)$. Homens altos recidivaram mais freqüentemente que mulheres e cessar o hábito tabágico diminuiu o risco de recorrência. A série estudada demonstra altos índices de recorrência após o tratamento inicial com drenagem pleural e confirma a impressão prática de que não existe uma definição sobre os potenciais fatores de risco para o desenvolvimento do segundo episódio de PEP, o que vale dizer: qualquer paciente, independente da apresentação clínico-radiológica, no primeiro episódio de PEP, ao ser tratado conservadoramente ou com drenagem pleural, possui um potencial de recorrência que varia entre 20 e $60 \%$.

$\mathrm{Na}$ tentativa de identificar uma população de pacientes que apresentasse maiores riscos para o desenvolvimento de recorrência do pneumotórax espontâneo, a tomografia computadorizada de tórax (TCT) surgiu como uma alternativa promissora para verificação de anormalidades pulmonares que sustentassem a indicação da abordagem cirúrgica. No entanto, os estudos sobre a utilização de TCT nos casos de pneumotórax espontâneo não demonstraram correlação entre os achados morfológicos, como número e tamanho de bolhas, blebs, bolhas contralaterais ou fibrose envolvendo o parênquima pulmonar, e os indices de recorrência. Nesse sentido, a TCT nos casos de PEP, como método orientador da indicação de cirurgia, não deve ser utilizada rotineiramente $e^{(13-15)}$.

Estabelecida a indicação de tratamento cirúrgico, impõe-se a questão: qual o melhor método?

Duas são as principais alternativas no que diz respeito ao acesso ao tórax: toracotomia axilar ou videotoracoscopia. A experiência inicial com a toracotomia axilar mostrou índices de recidiva inferiores a $1 \%$, assim como indices de complicações inferiores a $4 \%^{(10)}$. Deslauriers et al. ${ }^{(8)}$, num estudo de 409 pacientes submetidos à toracotomia axilar com ressecção de bolhas e pleurectomia apical, obtiveram índices de mortalidade de $0,2 \%$ (um paciente com tumor cerebral não identificado inicialmente) e complicações de 1,2\% (hemotórax e fuga aérea persistente). Toracotomia axilar com pleurodese abrasiva, em várias séries publicadas ${ }^{(8,9,27)}$, mostrou taxas de recidiva de 1 a 3,6\%, complicações de 5,9\% e mortalidade zero. A alternativa da sutura mecânica, acrescida ao método, propiciou redução no tempo operatório.

Naunheim et al. ${ }^{(16)}$ publicaram sua experiência com a videotoracoscopia no tratamento do pneumotórax espontâneo primário e secundário em 113 pacientes e 121 procedimentos. Índice de recidiva de $4,1 \%$ e $8 \%$ de complicações, que consistiram em fuga aérea prolongada por mais de cinco dias e determinaram reintervenção em dois pacientes. O período médio de internação foi de três dias e $84 \%$ dos pacientes receberam alta no $5^{\circ}$ dia de pósoperatório; acompanhamento médio desta série foi 13 meses.

A despeito de várias publicações sugerindo os benefício da videotoracoscopia ${ }^{(17-20)}$, o único trabalho prospectivo e randomizado, estabelecendo a comparação entre a toracotomia póstero-lateral e a videotoracoscopia foi a publicação de Waller et al. ${ }^{(21)}$. Mesmo com menores índices de recidiva para os submetidos à toracotomia $(3,3 \% \mathrm{x}$ $13 \%$ nos pacientes com PEP), os autores concluem sobre as vantagens da videotoracoscopia baseados no menor tempo de hospitalização e na menor intensidade da dor no pós-operatório. A avaliação das séries da literatura ${ }^{(22,23)}$ comparando os resultados da toracotomia axilar, em vez de toracotomia póstero-lateral, e a videotoracoscopia não demonstrou diferenças significativas no que diz respeito aos parâmetros utilizados por Waller et al.

Consideradas, atualmente, as opções cirúrgicas mais utilizadas para o tratamento do PEP, a toracotomia axilar $e$ a videotoracoscopia abrigam índices muito baixos de morbidade e mortalidade zero. Tal constatação não poderia permitir a discussão da sua utilização terapêutica no primeiro episódio de PEP, independente da forma de apresentação clínico-radiológica ${ }^{(24-26)}$ ? 
O PEP incide sobre uma população de pacientes jovens, teoricamente com menos riscos de submeter-se a um tratamento que envolva anestesia geral. A evolução das técnicas anestésicas e analgésicas foi evidente. O exemplo mais eloqüente é representado pelo tratamento de pacientes portadores de enfisema pulmonar e candidatos à cirurgia de redução de volume pulmonar. Teríamos os mesmos resultados verificados atualmente se dispuséssemos apenas dos recursos anestésicos, analgésicos e de terapia intensiva de que dispúnhamos há dez ou 20 anos?

Se podemos indicar cirurgia de ressecção pulmonar, com índices de morbidade e mortalidade aceitáveis, para esta população de pacientes pneumopatas graves, por que não oferecê-la aos pacientes portadores de PEP, que são, na subtotalidade dos casos, pacientes jovens e previamente hígidos? O risco anestésico não nos parece um argumento sustentado para ponderarmos a indicação da toracotomia axilar como primeira escolha no primeiro episódio de PEP.

\section{CONCLUSÃO}

A toracotomia axilar com pleurodese abrasiva mostrouse um método efetivo para o tratamento do pneumotórax espontâneo primário. Os resultados observados nesta série de 35 pacientes, no que se refere à morbidade, tempo de internação hospitalar e recidiva, são semelhantes aos verificados na literatura e comprovam a eficácia da técnica. A efetividade do método, quando comparados grupos de pacientes operados no primeiro ou segundo episódios de PEP, não mostrou diferença estatisticamente significante ( $p>0,005)$.

A evolução do trabalho médico, alicerçado no avanço tecnológico hoje evidenciado, impõe a necessidade de reavaliarmos condutas $e$ atitudes que possam estar em descompasso com este avanço. Uma nova abordagem na indicação cirúrgica do tratamento do pneumotórax espontâneo pode representar um exemplo dessa constatação.

\section{REFERÊNCIAS}

1. Gaensler E. Parietal pleurectomy for recurrent spontaneous pneumothorax. Surg Gynecol Obstet 1956;102:293-308.

2. Seremetis MG. The management of spontaneous pneumothorax. Chest 1970;57:65-68.

3. Dumont P, Diemont F, Massard G, Toumieux B, Wihlm JM, Morand G. Does thoracoscopic approach for surgical treatment for spontaneous pneumothorax represent progress? Eur J Cardiothorac Surg 1997; 11:27-31

4. Andrivet P, Djedaini K, Teboul JL, Brochard L, Dreyfuss D. Spontaneous pneumothorax. Comparison of thoracic drainage vs immediate or delayed needle aspiration. Chest 1995;108:335-339.

5. Schoenenberger RA, Haefeli WE, Weiss P. Timing of invasive procedures in therapy for primary and secondary spontaneous pneumothorax. Arch Surg 1991;126:764-766.
6. Jain SK, Al-Kattan KM, Hamdy MG. Spontaneous pneumothorax: determinants of surgical intervention. J Cardiovasc Surg (Torino) 1998; 39:107-111.

7. Paape K, Fry WA. Spontaneous pneumothorax. Chest Surg Clin North Am 1994;4:517-537.

8. Deslauriers J, Beaulieu M, Després JP, Lemieux M, Leblanc J, Desmeules M. Transaxillary pleurectomy for treatment of spontaneous pneumothorax. Ann Thorac Surg 1980;30:569-574.

9. Pinto $\mathrm{F}^{\circ} \mathrm{DR}$, Camargo JJP, Furian MB: Toracotomia axilar no tratamento cirúrgico do pneumotórax espontâneo. Anais do $\mathrm{I}^{\circ}$ Congresso Sul-americano de Cirurgia Torácica, Porto Alegre, 1992.

10. Massard G, Thomas P, Wihlm JM. Minimally invasive management for first and recurrent pneumothorax. Ann Thorac Surg 1998;66:592599.

11. Rhea JR, De Luca AS, Grene RE. Determining the size of pneumothorax in the upright patient. Radiology 1982;144:733-739.

12. Sadikot RT, Greene T, Meadows K, Arnold AG. Recurrence of primary spontaneous pneumothorax. Thorax 1997;52:805-809.

13. Warner BW, Bailey WW, Shipley RT. Value of computed tomography of the lung in the management of primary spontaneous pneumothorax. Am J Surg 1991;162:39-42.

14. Lesur O, Delorme N, Fromaget JM. Computed tomography in the etiologic assessment of idiopathic spontaneous pneumothorax. Chest 1990;98:341-347

15. Mittlehner W, Friedrich M, Dismann W. Value of computer tomography in the detection of bullae in the patients with primary spontaneous pneumothorax. Respiration 1992;59:221-227.

16. Naunheim KS, Mack MJ, Hazelrigg SR, Ferguson MK, Ferson PF, Boley TM, Landreneau RJ. Safety and efficacy of video-assisted thoracic surgical techniques for the treatment of spontaneous pneumothorax. J Cardiovasc Surg 1995; 109:1198-1203

17. Crisci R, Coloni GF. Video-assisted thoracoscopic surgery vs thoracotomy for recurrent spontaneous pneumothorax. A comparison of results and costs. Eur J Cardiothorac Surg 1996;10:556-560.

18. Weatherford DA, Stephenson JE, Taylor SM, Blackhurst D. Thoracoscopy versus thoracotomy: indications and advantages. Am Surg 1995; 61:83-86.

19. Atta HM, Latouf O, Moore JE, Caudill DR, Snyder AB. Thoracotomy vs video-assisted thoracoscopic pleurectomy for spontaneous pneumothorax. Am Surg 1997;63:209-212.

20. Horio H, Nomori H, Fuyuno G, Kobayashi R, Suemasu K. Limited axillary thoracotomy vs video-assisted thoracoscopic surgery for spontaneous pneumothorax. Surg Endosc 1998;12:1155-1158.

21. Waller DA, Forty J, Morrit GN. Video-assisted thoracoscopic surgery versus thoracotomy for spontaneous pneumothorax. Ann Thorac Surg 1994;58:372-377

22. Bertrand PC, Regnard JF, Spaggiari L, Levi JF, Magdeleinet P, Levasseur P. Immediate and long-term results after surgical treatment of primary spontaneous pneumothorax by VATS. Ann Thorac Surg 1996; 61:1641-1645.

23. Cole FH, Khandekar A, Maxwell JM, Pate JW, Walker WA. Videoassisted thoracic surgery: primary therapy for spontaneous pneumothorax. Ann Thorac Surg 1995;60:931-935.

24. Schramel FM, Sutedja TG, Braber JC, van Mourik JC, Postmus PE Cost effectiveness of video-assisted thoracoscopic surgery versus conservative treatment for first time or recurrent spontaneous pneumothorax. Eur Respir J 1996;9:1821-1825.

25. Passlick B, Born C, Thetter O. Cost comparison of minimal invasive surgery vs. standard operation exemplified by primary pneumothorax. Langenbecks Arch Chir Suppl Kongressbd 1997;114:1290-1292.

26. Baumann MH, Strange C. Treatment of pneumothorax. A more aggressive approach? Chest 1997;112:789-804. 